\title{
Observational study on the effects of a topical formulation in patients with premature ejaculation
}

KEY WORDS: Premature Ejaculation; Clove oil; Zanthoxylum Buongeanum; Aloe; Bisabolol; ejaculation.

Submitted 13 November 2020; Accepted 19 November 2020

To the Editor

Premature ejaculation (PE) has been defined as the inability to control or delay ejaculation, resulting in dissatisfaction or distress of the patient (1).

Although PE is the most frequent sexual dysfunction, it is still underdiagnosed. An accurate clinical history is the best diagnostic approach that, in the majority of cases, is enough to differentiate between primary and acquired PE. Nowadays, treatment is not curative but is effective in increasing the intravaginal ejaculatory latency time (IELT), improving the sexual satisfaction of the couple. Combination of behavioural techniques with pharmacotherapy is the best way of treatment.

Major pharmacological treatments of PE include selective serotonin reuptake inhibitors antidepressants (dapoxetine, paroxetine) and topical anesthetics (2).

From 2019 it has been introduced in the Italian market a new product for the topical natural approach of PE, containing clove oil, Zanthoxylum bungeanum fruit extract with aloe and bisabolol.

The aim of the present study was to evaluate the efficacy and the tolerability this topical formulation in patients of different age with PE.

An observational study was conducted in order to evaluate efficacy and tolerability of the topical formulation Endep ${ }^{\circledR}$ Spray in patients with premature ejaculation (PE).

Patients with clinical diagnosis of PE were enrolled in this observational study. All the patients underwent clinical evaluation including administration of Premature Ejaculation Diagnostic Tool (PDT) and International Index of Erectile Function (IIEF-5) questionnaires $(3,4)$.

The patients were assigned to use Endep ${ }^{\circledR}$ Spray (two puff before the sexual intercourse) for 30 days. The main outcome measure, evaluated by questionnaire, was the PEDT score; the secondary outcome was the improvement in the IIEF-5 score and the product tolerability evaluated along the whole study period. Eligible sunjects were $\geq 18$ and $\leq 75$ years of age with diagnosis of PE according to the European Association of Urology (EAU) guidelines. Patients with supposed or recognized intolerance towards one or more components of the product and patients with prostatic or urethral inflammatory and non-inflammatory pathology (prostatitis, urethritis) were excluded from the study. Endep ${ }^{\circledR}$ Spray contains clove oil, Zanthoxylum bungeanum fruit extract, aloe and bisabolol in the formulation of a sprayble hyperfluid emulsion. All patients were asked to apply one or two puff of the product in the penis area, 10 minutes before the sexual intercourse and were asked to massage the area till the product was completely absorbed.

Twenty-eight patients with diagnosis of PE were enrolled and treated in this observational study. PEDT and IIEF-5 scores were collected before and after the treatment with and data were analysed. The data show non normal distribution, therefore the Wilcoxon test was used.

At baseline visit (TO) and after 30 days of treatment (T30) patients were evaluated and asked to complete the two selfadministered questionnaires. At follow up examination after 30 days of treatment (T30) PEDT score showed a strong significant variation (median decrease $-3.5 ; \mathrm{p}=0.0002$ ). IIEF showed a significant but marginal variation (median increase +1.5$)$.

\section{Correlation with patients age and weight were checked by Pearson correlation test}

PEDT variation values showed a clear correlation with weight ( $\mathrm{p}=0.009)$; if weight was lower, the decrease in the PEDT score was greater. IIEF variation showed no correlation.

No correlation was found with the marital status (Wilcoxon test). All patients correctly used the product, showing a $100 \%$ compliance to the study protocol without any adverse effect.

The aim of the present study was to evaluate the efficacy of a sprayable hyperfluid emulsion in patients with premature ejaculation which has been recently marketed in Italy. The formula contains Clove oil, Zanthoxylum bungeanum fruit extract, Aloe, Bisabolol.

Zanthoxylum bungeanum Maxim is a natural desensitizer; the extract (Zanthalene) is obtained from the fruit of Sichuan pepper, a Chinese spice known for its chemestetic properties. In vitro investigations on a nerve-muscle preparation have shown that Zanthalene has a transitory action on the neuromuscolar synaptic transmission. This activation quick- 
ly leads to the depletion of the neurotransmitter. The action is mediated by the voltage-dependent $\mathrm{Na}+$ channels, by closing them at the suggested dosages and, in this way, by blocking the electric signal. Hydroxy- $\alpha$-sanshool has demonstrated in vivo to show an interesting activity on thermal and tactile sensitivity $(5,6)$. Eugenol is a volatile phenolic constituent of clove essential oil obtained from Eugenia caryophyllata buds and leaves. It is a functional ingredient of numerous products which have been used in the pharmaceutical, food and cosmetic industry. The wide range of eugenol activities includes antimicrobial, anti-inflammatory, analgesic and antioxidant (7).

Eugenol has anaesthetic properties, due to its ability to inhibit movement of sodium ions in peripheral nerves. Methyl eugenol is a potential candidate as an effective local anesthetic and analgesic. The antinociceptive and anesthetic effects of methyl eugenol result from the inhibitory action of methyl eugenol on peripheral $\mathrm{Na}+$ channels (8).

In the formulation Endep ${ }^{\circledR}$ Spray, which is a sprayable hyperfluid emulsion, the clove oil and the the Zanthoxylum bungeanum fruit extract are associated with aloe and bisabolol with soothing and emollient action .

In this observational study we evaluate the efficacy of the topical application of the product in patients with premature ejaculation. We found that the topical application on demand is able to improve PEDT score in patients with premature ejaculation aged between 18 and 75 years, without adverse events or reduction of the vaginal sensitivity of the female partner. However, this study shows some limitations related to the lack of a control group and the small number of patients; for this reason it can be considered as a preliminary observation for future studies and evaluations. In conclusion, in this observational study, we found that the topical treatment with Endep ${ }^{\circledR}$ Spray used before sexual intercourse significantly improves the PEDT score in patients with premature ejaculation.

Endep ${ }^{\circledR}$ Spray - IDI Integratori Dietetici Italiani, Italy

\title{
REFERENCES
}

1. Parnham A, Serefoglu EC. Classification and definition of premature ejaculation. Transl Androl Urol. 2016; 5:416-23.

2. Hu QB, Zhang D, Ma L, et al. Progresses in pharmaceutical and surgical management of premature ejaculation. Chin Med J (Engl). 2019; 132:2362-2372.

3. Althof SE. Patient reported outcomes in the assessment of premature ejaculation. Transl Androl Urol. 2016; 5:470-4.

4. Rosen RC, Riley A, Wagner G, et al. The international index of erectile function (IIEF): a multidimensional scale for assessment of erectile dysfunction. Urology. 1997; 49:822-30.

5. Bryant BP, Mezine I. Alkylamides that produce tingling paresthesia activate tactile and thermal trigeminal neurons. 1999. 25; 842:45260.

6. Lennertz RC, Makoto Tsunozaki, Bautista DM, Stucky CL. Physiological basis of tingling paresthesia evoked by hydroxy-alpha-sanshool. J Neurosci. 2010; 24; 30:4353-61.

7. Nejad SM, Özgünes H, Basaran N. Pharmacological and Toxicological Properties of Eugenol. Turk J Pharm Sci. 2017; 14:201-206.

8. Wang ZJ, Tabakoff B, Levinson SR, Heinbockel T. Inhibition of Nav1.7 channels by methyl eugenol as a mechanism underlying its antinociceptive and anesthetic actions. Acta Pharmacol Sin. 2015; 36:791-9.

Giuseppe Quarto, Luigi Castaldo, Giovanni Grimaldi, Alessandro Izzo, Raffaele Muscariello, Sisto Perdonà

\author{
Correspondence \\ Giuseppe Quarto, MD (Corresponding Author) \\ giuseppe.quarto@gmail.com \\ Luigi Castaldo, MD \\ Giovanni Grimaldi, MD \\ Alessandro Izzo, MD \\ Raffaele Muscariello, MD \\ Sisto Perdonà, MD \\ Division of Urology, IRCCS Fondazione G.Pascale, Naples (Italy)
}

